\title{
Image processing of concentrated and scattered objects
}

\author{
Vladimir V. Alekseev
}

E-mail:vvalex1961@mail.ru

\section{Denis V. Lakomov}

E-mail: LaDenV@yandex.ru

\section{Artem A. Shishkin}

E-mail: 68region333@mail.ru

\section{Ghassan AI Maamari}

E-mail: ghassan.almaamari@gmail.com

Tambov State Technical University

Address: 106, Sovetskaya Street, Tambov 392000, Russia

\begin{abstract}
In modern control systems and information processing, the recognition of objects in the image is complicated by the fact that the impact of negative factors introduces uncertainty into this process, leading to blurring of images. In this regard, it is necessary to develop models and algorithms that would reduce the degree of uncertainty in image processing. These models are necessary, for example, when monitoring environmentally hazardous objects, for search and detection of unauthorized burial of household waste, in the field of information security, in the analysis of x-rays and thermograms, in the actions of unmanned aerial vehicles of law enforcement agencies in autonomous mode. This article presents a description of information technology for recognition in the automated mode of objects in images. The basis of this technology is the algorithm of contour analysis of images. The main distinguishing feature of the algorithm is the use of convolution of the image in four directions, as well as the tracing procedure. The aim of the study was to develop algorithms for high-speed automated visualization of external objects. We present the results of the study of the algorithm of contour analysis in the processing of various images in the visible and infrared wavelengths. Recommendations are formulated for the choice of parameters of the contour analysis algorithm, such as the mean square deviation in image blur, minimum and maximum thresholds for filtering. The results of the study can be used in production management systems, life support of the city, technical vision, environmental conditions, monitoring of business processes, as well as in the creation of simulators for training operators of complex systems, etc. In addition, we show the expediency of applying the algorithm we developed in decision support systems.
\end{abstract}




\section{Graphical abstract}

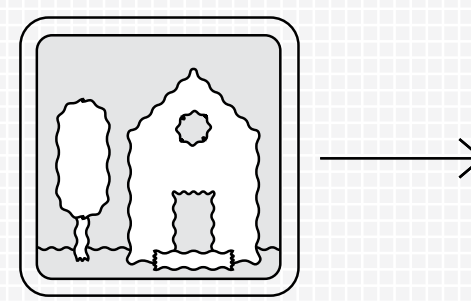

Original image

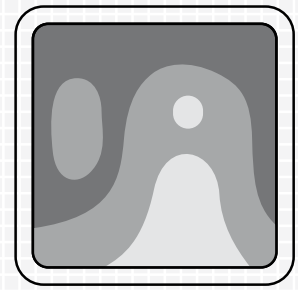

IR image

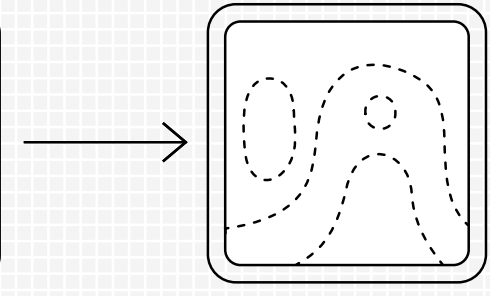

Contours of the object

Key words: detection; image; picture; contour analysis; algorithm; operator; uncertainty; object.

Citation: Alekseev V.V., Lakomov D.V., Shishkin A.A., Al Maamari G. (2019) Image processing of concentrated and scattered objects. Business Informatics, vol. 13, no 4, pp. 49-59.

DOI: $10.17323 / 1998-0663.2019 .4 .49 .59$

\section{Introduction}

A mid the acceleration of technological progress, management issues have become major concerns in terms of science, economy and public development. At the same time, it is clear that introducing new technologies into various areas of society is hard to overestimate. The expansion of management functions and problems in different walks of science, technology, economy, and health care outlines a transition towards multi-purpose management systems. Managing and making decisions in such systems can be implemented, although they are accompanied by enormous uncertainty related to undetermined properties of a complex management object or process. It is also related to uncertainty that comes from an external object, uncertainty of interconnection between management subsystems, uncertainty of management objectives, uncertainty of quality criteria, etc.

The aforementioned aspects of system development and management processes, especially when it comes to complex management systems, make it necessary to improve existing techniques and develop new ones. These new techniques will serve as tools for object detection in images and integrity violation detection purposes as well. Alternatively, they can be used to find damage in an object during photo or video analysis. Principles and practices of decision support systems have proven that not only are they effective tools for analyzing and monitoring complex and large systems (including business flow); they can also successfully serve as software for scalar replacement. Hence, all around the world research is being conducted aimed at improving and developing new methods and algorithms for decreasing the influence of uncertainty during image analysis.

A subject area analysis has concluded that the contour analysis algorithm developed by the authors on the basis of the Canny algorithm can be applied in decision support systems. It can be used in such systems to verify the pres- 
ence or absence of damage when objects, especially underground objects, are being monitored. That is why it is recommended to study the stages of the algorithm.

\section{Stages of the contour analysis algorithm}

A set of image pixels forms a matrix $\mathbf{P}$ :

$$
\mathbf{P}=\left(\begin{array}{ccc}
p_{11} & \cdots & p_{X 1} \\
\vdots & \ddots & \vdots \\
p_{1 Y} & \cdots & p_{X Y}
\end{array}\right),
$$

where $p_{i j}-$ the color value of an image pixel;

$X$ - the width of the image;

$Y$ - the height of the image.

Let $\mathbf{P}_{u}$ be a set specified by all elements of the pixel matrix $\left(\mathbf{P} \rightarrow \mathbf{P}_{u}\right)$.

Stage 1. Preliminary stage: converting to a grayscale image [1]. In order to convert an image to a grayscale image, the YUV model is needed. The model represents color in three variables: $Y$ is the brightness component and $U$ and $V$ are the auxiliary components for color restoration. A transition from the RGB model to the YUV model is obtained by the following formula:

$$
\begin{gathered}
Y=0.299 \cdot R+0.587 \cdot G+0.114 \cdot B \\
U=-0.14713 \cdot R-0.28886 \cdot G+0.426 \cdot B . \\
Y=0.615 \cdot R-0.51499 \cdot G-0.10001 \cdot B+128,
\end{gathered}
$$
where $R, G, B$ - the intensities of red, green, and blue, respectively [2].

Stage 2. Noise reduction. Noise reduction is a necessary step to remove side effects which occurred after image blurring. The developed algorithm uses image smoothing. A Gaussian function is applied in the algorithm as a smoothing filter.

Stage 3. Calculation of variables and defining the gradient directions. The gradient values can be calculated in pixels using a fourway method (vertical, horizontal, two diagonal ways). As a result, a one-to-one correspondence between a pixel and its value of the gradient brightness $\left(g_{i j}\right)$ is obtained.

The value of the gradient angle is rounded up in a way that the rounded-up value is multiple of 45 , thus it may be equal to $0,45,90$ or 135 degrees. The Robinson operator is used in the algorithm to compute the gradient value [3].

Stage 4. Pixel reduction using the gradient value that is different from the maximum value. Edge pixels are pixels that have the maximum gradient value (the local maximum) with respect to the neighboring pixels in accordance with the direction of the vector. Other pixels should be reduced. Their color characteristics in terms of RGB take the value of white color $(255,255,255)$ [4]. Further work deals with edge pixels. Let $\mathbf{C}$ be a set of edge pixels:

$$
\begin{gathered}
\mathbf{C}=\left\{\left\{p_{i j}\right\} \mid p_{i j} \neq(255,255,255)\right\}, \\
1 \leq i \leq X, 1 \leq j \leq Y,
\end{gathered}
$$

All other pixels should be grouped in a set $\mathrm{W}$ :

$$
\begin{gathered}
\mathbf{W}=\left\{\left\{p_{i j}\right\} \mid p_{i j}=(255,255,255)\right\}, \\
1 \leq i \leq X, 1 \leq j \leq Y, \\
\mathbf{C} \cup \mathbf{W}=\mathbf{P}_{u}
\end{gathered}
$$

Stage 5. Double detection of edges. In stage 4 all pixels, which are also the local maximums, must be verified to be consistent with two thresholds, $T_{\min }$ and $T_{\max }$. The threshold values are set by the user. If the gradient value of the pixel is lower than the minimum threshold, then the pixel belongs to set $\mathbf{W}$ and takes the white color value. Otherwise, if it is greater than the maximum threshold, then this pixel is contour and belongs to set $\mathbf{C}$. All other pixels that are left in-between the thresholds are going to be processed at the final stage [5].

The following formula yields a representation of this stage: 


$$
\left\{\begin{array}{l}
p_{i j} \in \mathbf{C}, \text { npu } g_{i j}>T_{\max }, \\
p_{i j} \in \mathbf{W}, n p u g_{i j}<T_{\min }, \\
p_{i j} \in \mathbf{N}, \text { npu } T_{\min }<g_{i j}<T_{\max },
\end{array}\right.
$$

where $\mathbf{N}$ - a set of pixels, the gradient values of which are located in-between the thresholds.

Correspondingly, expression (1) takes the following form:

$$
\mathbf{C} \cup \mathbf{W} \cup \mathbf{N}=\mathbf{P}_{u}
$$

Stage 6. Defining a stack of pixels between the threshold values. At the final stage, pixels belonging to set $\mathrm{N}$ are processed. The following condition must be satisfied for the pixels: if any neighboring pixel is an edge pixel, then the pixel that is being verified is considered an edge pixel as well. The main problem of this stage is to analyze all the pixels from set $\mathrm{N}$ in a way that groups of pixels which belong to the set and include edge pixels are also examined [6]. Pixels that don't turn out to be edge pixels take the value of white color. The distinctive characteristics of this stage are the following:

1. If the matrix element value of the current index is greater than the maximum threshold, then the pixel is applied to the stack of pixels. Hence, the vertex of the stack is then equal to 1 ;

2. The top element of the stack is removed. This entails subtracting 1 from its value;

3. Eight stack-derived indices, which are neighbors with respect to the index, are obtained;

4. The following condition for the neighboring indices must be verified: if the gradient value is greater than the minimum threshold and it hasn't been tagged 'considered,' then the index is tagged 'considered' and placed atop the stack;

Let us consider the most important stages in implementing the contour analysis algorithm.

\section{Noise reduction}

Image smoothing is the first step of the contour analysis algorithm. A Gaussian filter is applied to execute a procedure of smoothing, which blurs an image and allows us to reduce the influence of noise on the image analysis process. The main component of the Gaussian filter is an image kernel, also known as the convolution matrix, or mask. It is a matrix of odd size $(3 \times 3,5 \times 5,7 \times 7$, etc) for finding the central pixel of the matrix, where the Gaussian function is also applied. The function is called a weight function. A weight multiplier is a value that corresponds to its element of the convolution matrix. The weight function is the sum of all weight multipliers of the convolution matrix [7].

Image blurring is achieved by moving the convolution matrix along the pixel matrix of the image. The image is thereby convoluted in every position of the window or, in other words, the computation of a new value of every pixel using the values of the neighboring pixels takes place. The weight function is constant throughout the convolution process. The computation is performed as follows: the value of the respective pixel is multiplied by the coefficient of the weight function; after that the obtained products are summed. The final value is assigned to the pixel which is placed in the center of the convolution matrix [8].

The Gaussian function is also used to calculate the coefficients of the weight function per each coefficient. Eventually, the coefficient value is then placed in the respective element of the convolution matrix:

$$
f(x, y)=\frac{e^{-\frac{x^{2}+y^{2}}{2 \sigma^{2}}}}{2 \pi \sigma^{2}},
$$

where $x, y$ - the pixel's distance from the central pixel of the convolution matrix in the horizontal and vertical axis, respectively;

$$
\sigma-\text { the spread of the Gaussian function. }
$$


The values obtained for every pixel are then fixed in the respective elements of an intermediate matrix of the same size as the image. This additional intermediate matrix is necessary to avoid miscalculations that might be caused by the processed image pixels. It is important to mention threshold conditions. The conditions state that the corner pixels, unlike all other pixels, are deprived of any neighbors. To solve this problem, a temporary image should be created with the following size:

$$
x+\frac{R+1}{2}, y+\frac{R+1}{2},
$$

where $x, y-$ the length and heights of the image;

$R$ - the size of the convolution matrix.

The procedure to calculate values of the image analyzed is as follows:

1) A temporary image is created. The empty image is overlaid by the initial picture, while its corners are overlaid by the corner pixels of the initial image;

2) The temporary image is blurred;

3) An image from the temporary one is retrieved. It is to be of the same size as the processed image with regards to the center;

4) The color of the central pixel is achieved by summing the weight coefficients of the convolution kernel and the values of colors of the neighboring pixels of the image.

The values of the processed image pixels change and should be in consistency with the neighboring values.

Image smoothing decreases the noise level by aligning the pixel values with the neighboring dots. At this point we should also specify valid values for $\sigma$. The results of [9-13] allow us to draw the following inferences:

1) If we deal with the range where $0,1<\sigma<1$, then smoothing will be insignificant because the obtained pixel values, besides the central ones, are small enough to affect their colors;
2) If $1 \leq \sigma<10$, then the neighboring pixel values will be adjusted to other pixels. The convolution matrix has been applied to the other pixels before, therefore a major part of the noise will be removed;

3) Two-digit values of $\sigma$ enable blurring. This kind of blurring makes the noise and some pixels disappear from the image;

4) If $\sigma$ is much smaller than 1 , then there will be only the central pixel of the matrix that differs from zero. Blurring also will not occur.

\section{Finding the gradient values}

An operator for finding the gradient value in image pixels is the main feature of the contour analysis algorithm for concentrated and scattered objects. For further work, the direction and value of the gradient are required.

Let us consider a $3 \times 3$ matrix $\mathbf{D}$ containing the brightness values of the surroundings of the pixel:

$$
\mathbf{D}=\left[\begin{array}{lll}
d_{1} & d_{2} & d_{3} \\
d_{4} & d_{5} & d_{6} \\
d_{7} & d_{8} & d_{9}
\end{array}\right]
$$

The following formula yields the gradient value in every pixel in an image comprising concentrated or scattered objects [14]:

$$
G=\sqrt{G_{x}^{2}+G_{y}^{2}},
$$

where $G$ - the gradient value in a pixel $d_{5}$;

$G_{x}, G_{y}$ - approximate derivatives of the gradient value in terms of $x$ and $y$, respectively (take forms of two matrices).

Let us review existing math operators.

The Roberts cross operator. This operator facilitates high-speed calculations, although it has one disadvantage, i.e. noise sensitivity. Contour lines that can be obtained using such operator are thinner than those obtained by other operators. 
A matrix of approximate derivatives in accordance with the Roberts cross operator in $d_{5}$ for $G_{x}$ and $G_{y}$ can be computed in the following way:

$$
G_{x}=d_{9}-d_{5}, G_{y}=d_{8}-d_{6} .
$$

As the formula points out, the process of convolution can be performed in two possible ways, in a vertical or horizontal direction [15]:

$$
R b_{0}=\left[\begin{array}{cc}
-1 & 0 \\
0 & 1
\end{array}\right], R b_{1}=\left[\begin{array}{cc}
0 & -1 \\
1 & 0
\end{array}\right] .
$$

The Prewitt operator. Unlike the previous one, this operator is based on the use of $3 \times 3$ masks and also considers 8 directions of the gradient. However, only the straight directions are used, since they bring about the best results:

$$
p_{0}=\left[\begin{array}{ccc}
-1 & -1 & -1 \\
0 & 0 & 0 \\
1 & 1 & 1
\end{array}\right], p_{1}=\left[\begin{array}{ccc}
-1 & 0 & 1 \\
-1 & 0 & 1 \\
-1 & 0 & 1
\end{array}\right] .
$$

The matrix of approximate derivatives in accordance with the Prewitt operator for $G_{x}$ and $G_{y}$ can be calculated as follows:

$$
\begin{aligned}
& G_{x}=\left(d_{7}+d_{8}+d_{9}\right)-\left(d_{1}+d_{2}+d_{3}\right), \\
& G_{y}=\left(d_{3}+d_{6}+d_{9}\right)-\left(d_{1}+d_{4}+d_{7}\right) .
\end{aligned}
$$

The primary benefit of the Prewitt operator is an ability to reduce sensitivity to noise by using an augmented mask [16]:

The Sobel operator. This operator works with values that are approximate to their derivatives. This aids in detecting contours in areas where the gradient is a large number. The operator comprises two matrices. The difference between the second and first matrices is that the former is rotated by 90 degrees, so is it in the Prewitt operator. In order to reduce the blurring effect, a weight coefficient that is equal to 2 is used for the matrix elements. It is achieved by raising the influence of middle pixels to avoid losing the contours of images.

This operator resembles the Roberts cross operator and the Prewitt operator. The masks of the Sobel operator have the following form:

$$
S_{0}=\left[\begin{array}{ccc}
-1 & -2 & -1 \\
0 & 0 & 0 \\
1 & 2 & 1
\end{array}\right], S_{1}=\left[\begin{array}{ccc}
-1 & 0 & 1 \\
-2 & 0 & 2 \\
-1 & 0 & 1
\end{array}\right] .
$$

The matrix of approximate derivatives in accordance with the Sobel operator for $G_{x}$ and $G_{y}$ can be calculated as follows:

$$
\begin{aligned}
& G_{x}=\left(d_{7}+2 d_{8}+d_{9}\right)-\left(d_{1}+2 d_{2}+d_{3}\right), \\
& G_{y}=\left(d_{3}+2 d_{6}+d_{9}\right)-\left(d_{1}+2 d_{4}+d_{7}\right) .
\end{aligned}
$$

The fact that the Sobel operator has a rough approximation of the gradient, which leads to a great deal of contours being lost, serves as a drawback [17].

The Kirsch and Robinson operators. These operators consist of 8 symmetric masks. For instance, the center of the symmetry of the Robinson operator is a central axis containing only zeros. It takes just computing the values of the first four masks, meanwhile the others can be obtained by inversing the first ones. The operators are quite similar; however they differ in their complexities. The Kirsch operator uses weight coefficients, such as -3 and 5, while the Robinson operator deals with $-2,-1,0,1$ and 2 .

The masks of the Kirsch operator are as follows:

$$
\begin{aligned}
& k_{0}=\left[\begin{array}{ccc}
-3 & -3 & 5 \\
-3 & 0 & 5 \\
-3 & -3 & 5
\end{array}\right], k_{1}=\left[\begin{array}{ccc}
-3 & 5 & 5 \\
-3 & 0 & 5 \\
-3 & -3 & -3
\end{array}\right], \\
& k_{2}=\left[\begin{array}{ccc}
5 & 5 & 5 \\
-3 & 0 & -3 \\
-3 & -3 & -3
\end{array}\right], k_{3}=\left[\begin{array}{ccc}
5 & 5 & -3 \\
5 & 0 & -3 \\
-3 & -3 & -3
\end{array}\right], \\
& k_{4}=\left[\begin{array}{ccc}
5 & -3 & -3 \\
5 & 0 & -3 \\
5 & -3 & -3
\end{array}\right], k_{5}=\left[\begin{array}{ccc}
-3 & -3 & -3 \\
5 & 0 & -3 \\
5 & 5 & -3
\end{array}\right], \\
& k_{6}=\left[\begin{array}{ccc}
-3 & -3 & -3 \\
-3 & 0 & -3 \\
5 & 5 & 5
\end{array}\right], k_{7}=\left[\begin{array}{ccc}
-3 & -3 & -3 \\
-3 & 0 & 5 \\
-3 & 5 & 5
\end{array}\right] .
\end{aligned}
$$


Masks $k_{2}$ and $k_{6}$ correspond to the horizontal edge, masks $k_{0}$ and $k_{4}$ correspond to the vertical one, masks $k_{1}, k_{3}, k_{5}$ and $k_{7}$ correspond to the diagonal edge.

The main mask is the one that assists with obtaining the maximum value of $d_{5}$. This value is the gradient value of the pixel [18].

The Kirsch and Robinson operators are based on using just one mask which is rotated in the 8 compass directions: North, North West, West, South West, South, South East, East, North East. The masks of the Robinson operator have the following forms:

$$
\begin{aligned}
& r_{0}=\left[\begin{array}{lll}
-1 & 0 & 1 \\
-2 & 0 & 2 \\
-1 & 0 & 1
\end{array}\right], r_{1}=\left[\begin{array}{ccc}
0 & 1 & 2 \\
-1 & 0 & 1 \\
-2 & -1 & 0
\end{array}\right], \\
& r_{2}=\left[\begin{array}{ccc}
1 & 2 & 1 \\
0 & 0 & 0 \\
-1 & -2 & -1
\end{array}\right], r_{3}=\left[\begin{array}{ccc}
2 & 1 & 0 \\
1 & 0 & -1 \\
0 & -1 & -2
\end{array}\right], \\
& r_{4}=\left[\begin{array}{ccc}
1 & 0 & -1 \\
2 & 0 & -2 \\
1 & 0 & -1
\end{array}\right], r_{5}=\left[\begin{array}{ccc}
0 & -1 & -2 \\
1 & 0 & -1 \\
2 & 1 & 0
\end{array}\right], \\
& r_{6}=\left[\begin{array}{ccc}
-1 & -2 & -1 \\
0 & 0 & 0 \\
1 & 2 & 1
\end{array}\right], r_{7}=\left[\begin{array}{ccc}
-2 & -1 & 0 \\
-1 & 0 & 1 \\
0 & 1 & 2
\end{array}\right] .
\end{aligned}
$$

The gradient value in each pixel is rounded up and then set to the maximum value, which is calculated by using the masks. The angle between the rows of zeros in the mask turns out to be the angle of the gradient vector. The mask allows us to obtain the maximum gradient value [19].

The Laplace operator. It was first published in 1982. This algorithm facilitates the calculation of the second derivative using the formula:

$$
\Delta^{2} f=\frac{d^{2} f}{d x^{2}}+\frac{d^{2} f}{d y^{2}} .
$$

The operator is performed in two stages. In the first one, image smoothing takes place using the Gaussian filter. The second one involves finding the Laplace operator, which results in the appearance of double contours. The contours can be detected by approximating their values. Such approximation implies finding zeros at the point of intersection of the double contours. The masks of the Laplace operator have the following form:

$$
l_{0}=\left[\begin{array}{ccc}
0 & -1 & 0 \\
-1 & 4 & -1 \\
0 & -1 & 0
\end{array}\right], \quad l_{1}=\left[\begin{array}{ccc}
-1 & -1 & -1 \\
-1 & 8 & -1 \\
-1 & -1 & -1
\end{array}\right]
$$

The primary feature of the Laplace operator is the ability to find every pixel within or outside the double contour obtained by the Gaussian filter and Laplace operator [20].

Reference [21] incorporates the results of using all the aforementioned operators for analyzing an image. The authors have conducted an in-depth analysis of image processing results. The results indicate that the Prewitt, Robinson, Sobel operators bring out different results, yet they also complement one another. The Laplacian of Gaussian has demonstrated almost identical results for main contours, although false objects appeared as well, such as walls and a lawn in front of a building. In addition, the Kirsch and Robinson operators, unlike the others, have detected the greatest number of true contours in an image $[4,12,14]$.

\section{Test runs of the contour analysis algorithm}

The suggested information system for image processing of concentrated and scattered objects incorporates an operating system, database of images, the contour analysis algorithm, a linguistic and logical model to choose the algorithm parameters, image processing results comparison model, and cross-platform software to ensure interpretation of results.

A series of test runs of the software has been carried out.

The Robinson operator was used to determine the gradient. The values are $T_{\min }=20$, 
$T_{\max }=45$. These values (stage 5) enable us to obtain the best results in terms of the signalnoise ratio. Figures $1-3$ depict the results of image processing on the basis of the algorithm so developed.

The conducted research shown in Figures 4-5 demonstrates that the contour analysis algorithm shows a 10-15\% increase in detecting edge pixels compared to the standard Canny algorithm when it comes to image processing of concentrated and scattered objects. It is worth noting that the time needed to execute the algorithm has not increased dramatically. The number of test-run materials is 110 images.

\section{Recommendations on choosing the algorithm parameters}

The algorithm software allows us to detect objects in blurred or sharply-defined images and recognize them easily. The effectiveness of such research may be affected by the quality of an image captured by an IR device, distance range, camera angle, weather conditions. Unwanted sources of heat, i.e. residential buildings, technical gadgets, living beings, may hamper the analysis of an image.

The recommended parameters for an anal-

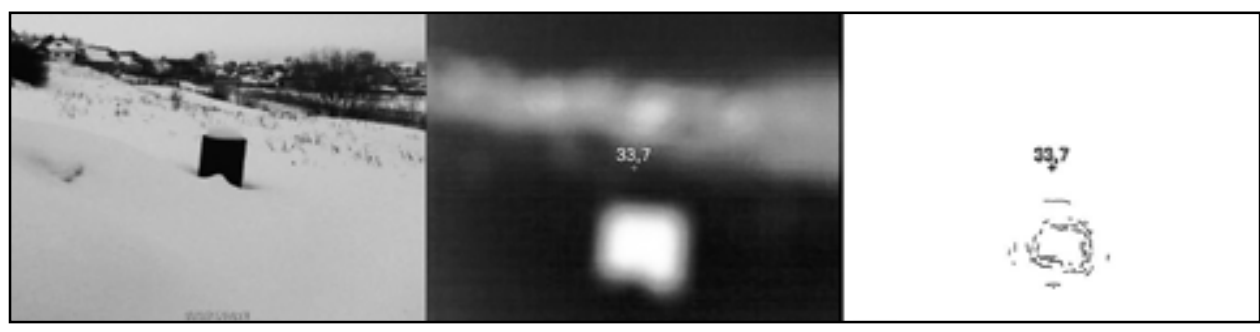

Fig. 1. Image processing of a vent unit

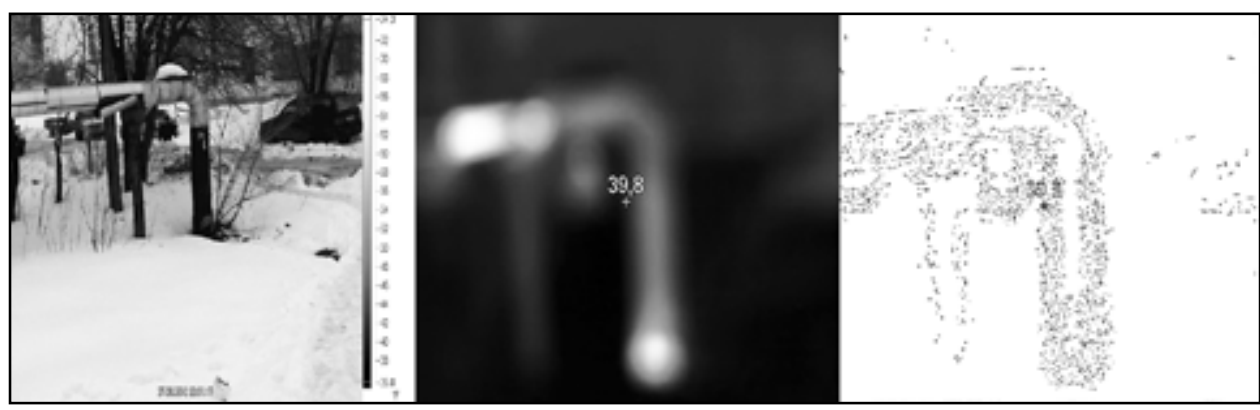

Fig. 2. Image processing of a heat pipeline

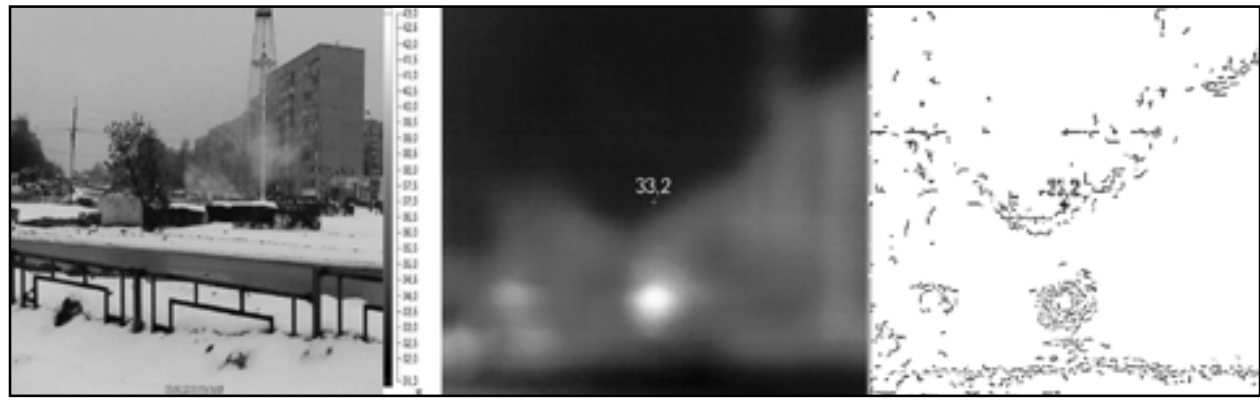

Fig. 3. Image processing of a manhole 


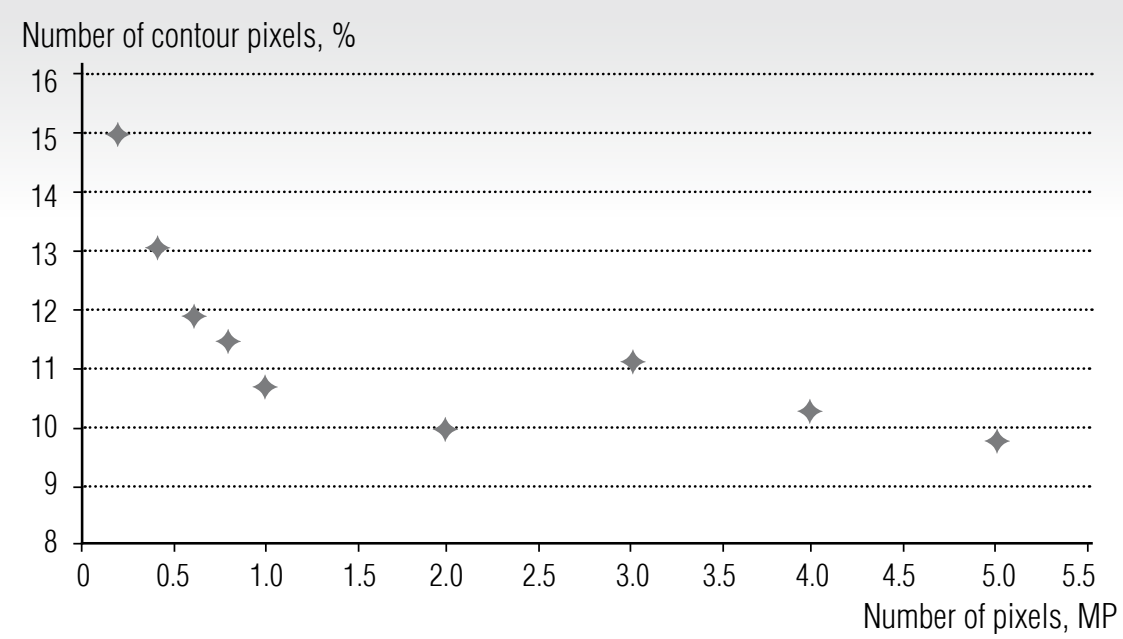

Fig. 4. The increase of the number of detected pixels if the algorithm is used in comparison with the Canny algorithm

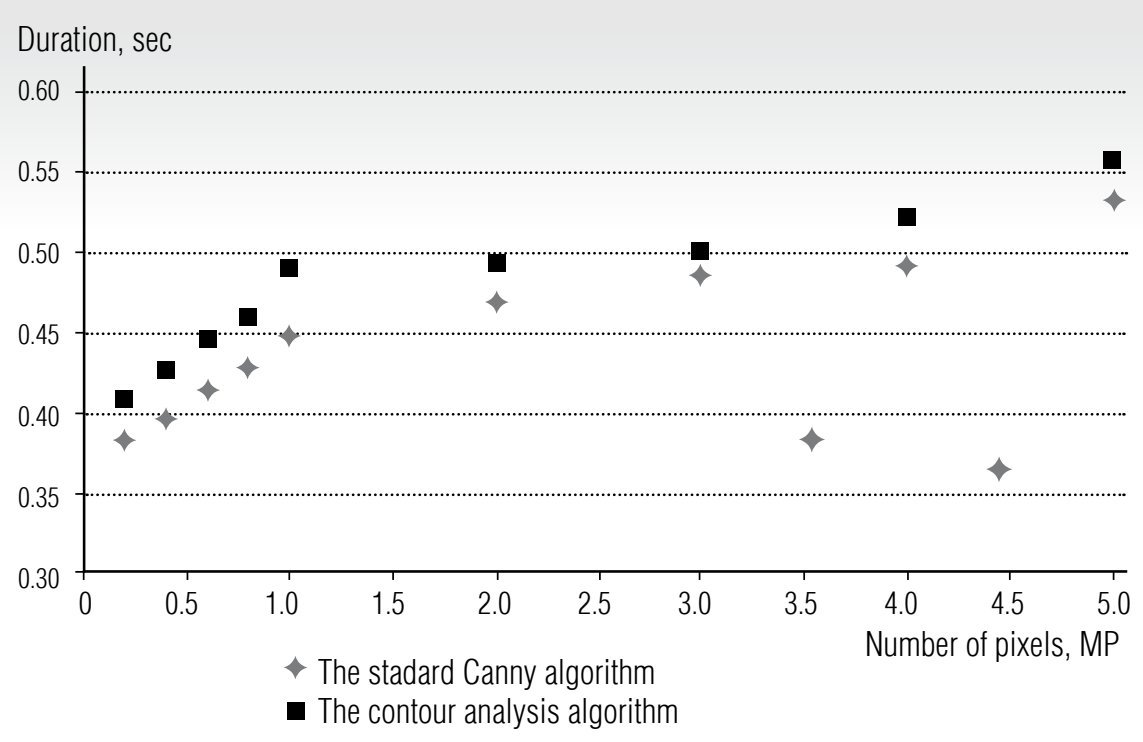

Fig. 5. The elapsed time of the algorithms to process images

ysis of a sharply-defined image are $T_{\min }=20$, $T_{\max }=45, \sigma=1$.

The recommended parameters for an analysis of a blurred image are $T_{\min }=5, T_{\max }=15$, $\sigma=0.01$.

The recommended parameters are applicable for the considered and similar types of images. The linguistic and logical model is being developed to choose the algorithm parameters that depend on image specifications.
The advantages of the algorithm are the following:

$\downarrow$ an increase in the number of detected edge pixels;

$\checkmark$ minimization of contour fragmentation;

a decrease of image-noise-based uncertainty by virtue of the Gaussian filter.

The lack of well-defined criteria of choosing the threshold values which leads to the dis- 
tortion of true contours and the appearance of false ones is the main drawback of the algorithm.

The research results suggest that the algorithm software should be improved to make the image corners less rounded since this causes the edges to be removed.

\section{Conclusion}

This paper showcases the description of an image processing technology for concentrated and scattered objects on the basis of the contour analysis algorithm.

The algorithm operates using the Robinson operator and the process of convolution applied to an object in four directions. It ensures that contour fragmentation is minimized.

We have provided the research results of the contour analysis algorithm for image processing of different objects in the infrared range. Recommendations on choosing the algorithm parameters are suggested on the basis of the research.

The research results may find application in technical vision systems designed to detect damage and monitor analyzed objects. Additionally, sharply-defined and scattered objects monitoring systems may see a benefit of the algorithm by training new operators. Furthermore, it can be highly useful for decision support systems and detecting integrity violations in sharply-defined and scattered objects.

\section{References}

1. Huo X.Q., Zheng W.L., Lu B.L. (2016) Driving fatigue detection with fusion of EEG and forehead EOG. Proceedings of the International Joint Conference on Neural Networks (IJCNN), Vancouver, BC, Canada, 24-29 July 2016, pp. 897-904. DOI: 10.1109/IJCNN.2016.7727294.

2. Alekseev V.V., Gromov Yu.Yu., Gubskov Yu.A., Ishchuk I.N. (2014) Methodology of remote assessment of spatial distributions of optical-thermal parameters of the objects disguised under the surface. Moscow: Nauchtehlitizdat (in Russian).

3. Karasev P.I., Gubskov Yu.A. (2015) Processing of graphic images in video surveillance systems. Herald of the Voronezh Institute FSIN of Russia, no 2, pp. 35-37 (in Russian).

4. Alekseev V.V., Lakomov D.V. (2017) Robinson Operator and its application in the canny algorithm for image recognition under uncertainty. Proceedings of the XV All-Russian Scientific Conference "Neurocomputers and Their Application", 14 March 2017. Moscow: MSUPE, pp. 89-90 (in Russian).

5. Gonzalez R., Woods R. (2005) Digital image processing. Moscow: M: Technosphere (in Russian).

6. Pytyev Yu.P. (2010) Methods of morphological image analysis. Moscow: FIZMATLIT (in Russian).

7. Potapov A.A. (2008) The latest methods of image processing. Moscow: FIZMATLIT (in Russian).

8. Srivastava S., Delp E.J. (2003) Video-based real-time surveillance of vehicles. Journal of Electronic Imaging, vol. 22, no 4, 041103. DOI: 10.1117/1.JEI.22.4.041103.

9. Furman Ya.A. (2003) Introduction to contour analysis. Applications to image and signal processing. Moscow: FIZMATLIT (in Russian).

10. Basarab M.A., Volosyuk V.K., Goryachkin O.V., Zelensky A.A. (2007) Digital signal and image processing in radiophysical applications. Moscow: FIZMATLIT (in Russian).

11. Canny J. (1986) A computational approach to edge detection. IEEE Transactions on Pattern Analysis and Machine Intelligence, vol. PAMI-8, no 6, pp. 679-698.

12. Kim N.V., Krylov I.G. (2012) Group application of unmanned aerial vehicle in surveillance tasks. Trudy MAI, no 62. Available at: https://mai.ru/upload/iblock/bbb/gruppovoe-primenenie-bespilotnogoletatelnogo-apparata-v-zadachakh-nablyudeniya.pdf (accessed 15 April 2019) (in Russian).

13. Alekseev V.V., Karasev P.I., Lakomov D.V. (2016) Analysis of image processing methods applicable in the conditions of uncertainty. Proceedings of the XVI International Conference "Informatics: problems, methodology, technologies, ”Voronezh, 11-12 February 2016, pp. 37-41 (in Russian). 
14. Alekseev V.V., Lakomov D.V. (2016) Analysis of the applicability of blur in image recognition under uncertainty. Proceedings of the III International Scientific and Practical Conference "Virtual modeling, prototyping and industrial design,” Tambov, Russia, 17-19 November 2015, vol. 2, pp. 138-141 (in Russian).

15. Kim N.V., Kuznetsov A.G., Krylov I.G. (2010) Application of vision systems on unmanned aerial vehicles in the tasks of orientation on the ground. Aerospace MAI Journal, vol. 17, no 3, pp. 46-49 (in Russian).

16. Kanishka Madusanka D.G., Gopura R.A.R.C., Amarasinghe Y.W.R.; Mann G.K.I. (2017) Hybrid vision based reach-to-grasp task planning method for trans-humeral prostheses. IEEE Access, vol. 5 , no 99, pp. 16149 -16161. DOI: 10.1109/access.2017.2727502.

17. Jain A., Abbas B., Farooq O., Garg S.K. (2016) Fatigue detection and estimation using auto-regression analysis in EEG. Proceedings of the 2016 International Conference on Advances in Computing, Communications and Informatics (ICACCI), Jaipur, India, 21-24 September 2016, pp. 1092-1095. DOI: 10.1109/ICACCI.2016.7732190.

18. Yang K.F., Li C.-Y., Li Y.-J. (2014) Multifeature-based surround inhibition improves contour detection in natural images. IEEE Transaction on Image Processing, vol. 23, no 12, pp. 5020-5032. DOI: 10.1109/TIP.2014.2361210.

19. Yang K.-F., Gao S.-B., Guo C.-F., Li C.-Y., Li Y.-J. (2015) Boundary detection using doubleopponency and spatial sparseness constraint. IEEE Transaction on Image Processing, vol. 24, no 8. pp. 2565-2578. DOI: 10.1109/TIP.2015.2425538.

20. Muthukrishnan R., Radha M. (2012) Edge detection techniques for image segmentation. International Journal of Computer Science \& Information Technology, vol. 3, no 6, pp. 259-267. DOI: 10.5121/ ijcsit.2011.3620.

21. Guan T., Wang Y., Duan L., Ji R. (2015) On-device mobile landmark recognition using binarized descriptor and multifeature fusion. ACM Transactions on Intelligence Systems Technology, vol. 7, no 1, article 12. DOI: $10.1145 / 2795234$.

\section{About the authors}

\section{Vladimir V. Alekseev}

Dr. Sci. (Tech.), Professor;

Head of the Department "Information systems and information security",

Tambov State Technical University,

106, Sovetskaya Street, Tambov 392000, Russia;

E-mail: vvalex1961@mail.ru

\section{Denis V. Lakomov}

Doctoral Student, Department "Information systems and information security", Tambov State Technical University,

106, Sovetskaya Street, Tambov 392000, Russia;

E-mail: LaDenV@yandex.ru

\section{Artem A. Shishkin}

Doctoral Student, Department "Information systems and information security”, Tambov State Technical University, 106, Sovetskaya Street, Tambov 392000, Russia;

E-mail: 68region333@mail.ru

\section{Ghassan Al Maamari}

Doctoral Student, Department "Information systems and information security", Tambov State Technical University,

106, Sovetskaya Street, Tambov 392000, Russia;

E-mail: ghassan.almaamari@gmail.com 